\title{
Indentation modulus extrapolation and thickness estimation of ta-C coatings from nanoindentation
}

\author{
L. Lorenz ${ }^{1,2, *}$ (D) T. Chudoba ${ }^{3}$, S. Makowski ${ }^{2}$, M. Zawischa ${ }^{2}$, F. Schaller ${ }^{2}$, and \\ V. Weihnacht ${ }^{2}$ \\ ${ }^{1}$ Institute of Manufacturing Technology, Technische Universität Dresden, Dresden, Germany \\ ${ }^{2}$ Fraunhofer Institute for Material and Beam Technology IWS, Dresden, Germany \\ ${ }^{3}$ ASMEC Advanced Surface Mechanics GmbH, Dresden, Germany
}

Received: 7 February 2021

Accepted: 3 August 2021

Published online:

3 September 2021

(C) The Author(s) 2021

\begin{abstract}
Coatings used in tribological applications often exhibit high hardness and stiffness to achieve high wear resistance. One coating characterization method frequently used is nanoindentation which allows the determination of indentation hardness and indentation modulus among other material properties. The indentation modulus describes the elastic surface behavior during indentation and is, among hardness, a direct indicator for wear resistance. To obtain the true indentation modulus of a coating, it must be measured with varying loads and then extrapolated to zero load. Current recommendation of the standard ISO 14577-4:2016 is a linear extrapolation which fits poorly for nonlinear curves. Such nonlinear curves are commonly found for high hardness mismatches between coating and substrate, for example, superhard tetrahedral amorphous carbon coatings (ta-C) on a steel substrate. In this study, we present a new empirical fit model, henceforth named sIGMOID. This fit model is compared to several existing fit models described in the literature using a large number of nanoindentation measurements on ta-C coatings with wide ranges of indentation modulus and coating thickness. This is done by employing a user-independent and model agnostic fitting methodology. It is shown that the sigmoid model outperforms all other models in the combination of goodness of fit and stability of fit. Furthermore, we demonstrate that the sigmoid model's fit parameter directly correlates with coating thickness and thus allows for a new approach of determining ta-C coating thickness from nanoindentation.
\end{abstract}

Handling Editor: Avinash Dongare.

Address correspondence to E-mail: lars.lorenz@tu-dresden.de 


\section{Introduction}

\section{Nanoindentation}

Usually, the indentation modulus $E_{I T}$ can be reliably measured with the Oliver-Pharr method from the unloading curve [1]. For coated materials, a correct measurement of $E_{I T}$ is hindered by the substrate's influence. The elastic deformation field has a theoretically unlimited range, and even sample areas far away from the indent position have an influence on the indentation depth. Therefore, unlike hardness it is not possible to give a limit where the substrate influence can be neglected. For this reason, the indention standard ISO 14577 part 4 [2] describes a method how the pure $E_{I T}$ of the coating can be obtained. It requires a series of indention measurements with different forces and depths and the extrapolation of the function $E_{I T}(a)$ or the normalized function $E_{T T}\left(a / t_{c}\right)$ to zero depth with $a$ as contact radius and $t_{c}$ as coating thickness. According to the standard [2], a minimum of 10 indents should be performed per force and thus at least 50 measurements are required. This is a relatively time-consuming procedure; therefore, other methods have been developed:

- Cyclic load-unload measurement,

- Continuous stiffness measurement,

- Quasi-continuous stiffness measurement.

In the standard, a linear fit is recommended for simplicity but also nonlinear fit methods are permitted.

\section{Cyclic load-unload measurement}

In cyclic load-unload measurements (also called load/partial unload), the contact stiffness can be determined for every unload cycle and give therefore as many modulus results over depth as cycles. The analysis is done in the same way as single load-unload measurements. The method reduces the overall measurement time and number of measuring points, while the technique is realizable with nearly every nanoindentation system. However, the test time per test position is still long and therefore the thermal drift of the instrument has a larger influence and needs to be corrected. In case of a nonlinear thermal drift, the accuracy is reduced, especially for the first cycles at low loads.

\section{Continuous stiffness measurement}

The continuous stiffness measurement (CSM) method superimposes a small harmonic oscillation $\delta F$ to the loading force. By measuring the displacement oscillation $\delta h$ and the phase angle between force and displacement oscillation, one can obtain the contact stiffness for any point on the load-displacement curve and therefore also the depth-dependent $E_{I T}$. The method was patented in 1989 by Oliver and Pethica [3] and thus for a long time only available for one instrument type. Today, the patent has expired and most nanoindentation instruments offer dynamic test capabilities. The disadvantage of the method is that only a few oscillation data points are available for every point in the curve and that, due to the quasistatic loading, any oscillation ends at a slightly higher force level than its start level and the oscillation cycle is not fully complete. However, the application of this method was a large progress regarding the extraction of the correct modulus for thin coatings.

\section{Quasi-continuous stiffness measurement}

The quasi-continuous stiffness measurement (QCSM) method was developed by Chudoba as an alternative to the CSM method. Here, the load is increased in a stepwise manner without any oscillation, and the oscillation is only switched on during a short dwell time after every load step as shown in Fig. 1 . This has the advantage that the feedback during the force application is not influenced by any oscillation and

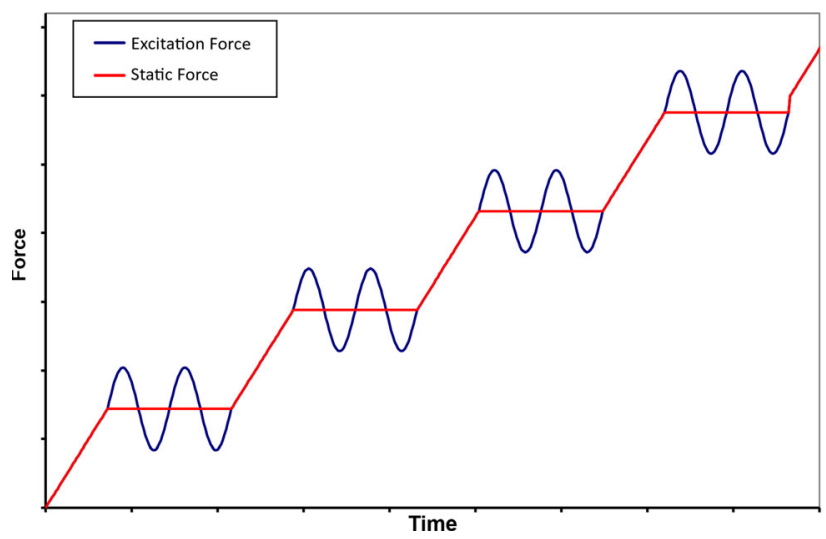

Figure 1 Load progression during QCSM. The static load progression is stopped shortly, and a sinusoidal excitation force is superimposed. The contact stiffness is calculated similarly to CSM. 
that force controllability is improved. During the dwell time of $0.5-3 \mathrm{~s}$ (depending on frequency), the average force is constant and oscillations can be averaged easily because the oscillation cycle is always finished at the same force level. This method has therefore a smaller measurement error than both CSM and the cyclic load-unload measurement method and the scatter of the results is small-down to a few-nanometer indentation depth. Further, a very good agreement to test results of conventional load-unload cycles could be achieved without additional corrections. The typical number of 30-40 points in one load segment is smaller than for the CSM method but larger than for cyclic measurements. It is also high enough for a good presentation of the depth dependency of the modulus for coated materials.

\section{Models for fitting indentation modulus- displacement curves}

Most of the alternative models for fitting the $E_{I T}-a$ data can be grouped into two basic forms: linear additive Eq. (1) and reciprocal additive Eq. (2).

$E_{I T}=E_{I T, 1}+\left(E_{I T, 2}-E_{I T, 1}\right) \cdot \Phi_{l}$

$\frac{1}{E_{I T}}=\frac{1}{E_{I T, 1}}+\left(\frac{1}{E_{I T, 2}}-\frac{1}{E_{I T, 1}}\right) \cdot \Phi_{r}$

$\Phi$ is the model-specific weight function which is set up to approach the coating indentation modulus $E_{I T, c}$ for $a=0$ and the substrate indentation modulus $E_{I T, S}$ for $a \rightarrow \infty$. Accordingly, $E_{I T, 1}$ and $E_{I T, 2}$ describe model-dependent substrate and coating indentation moduli. $k$ is an additional fit factor.

The fit models listed in Table 1 were compiled and modified from the literature. Models dependent on $h$ have been changed to a function of $a$ where necessary to achieve independency from the indenter geometry. ExPONENTIAL and ReCIPROCAL ExPONENTIAL use empirical $\Phi$. These fit models have first been described by Menčík et al. for fitting $E_{I T}-a$ curves [4]. The other models follow theoretical considerations.

LINEAR describes the linear fit according to ISO 14577-4:2016 [2].

$\mathrm{GAO}_{1}$ and $\mathrm{GAO}_{2}$ were originally based on thoughts by Gao et al. on the elastic indentation behavior of a flat, cylindrical punch into a solid surface [5]. $\mathrm{GAO}_{2}$ has later successfully been applied to a pyramidal indenter by Menčík et al. without modification [4].
PuCHI-CABRERA has first been proposed by Antunes et al. who transformed the linear additive form of $\mathrm{GAO}_{2}$ into a reciprocal additive one while keeping $\Phi$ [6]. The listed version with a modified $\Phi$ has been published by Puchi-Cabrera et al. [7].

Bull has been proposed by Bull according to his geometrical consideration of the loading of a coated substrate [8]. He theorizes the development of a supporting volume beneath the contact which is shaped like a truncated cone. Originally, it has been formulated with substrate thickness $t_{s}$ and coating thickness $t_{c}$ as follows:

$$
\begin{aligned}
E_{I T}= & {\left[E_{I T, c}^{-1}\left(1-\frac{\pi a}{\pi a+2 t_{c}}\right)\right.} \\
& \left.+E_{I T, s}^{-1}\left(\frac{\pi a}{\pi a+2 t_{c}}-\frac{\pi a}{\pi a+2\left(t_{c}+t_{s}\right)}\right)\right]^{-1}
\end{aligned}
$$

For the investigated samples, $t_{s} \gg t_{c}$ holds true, so the last term can be truncated:

$\frac{\pi a}{\pi a+2 t_{c}}-\frac{\pi a}{\pi a+2\left(t_{c}+t_{s}\right)} \approx \frac{\pi a}{\pi a+2 t_{c}}$

Additionally, $t_{c}$ is assigned as a second fit parameter.

LINEAR, EXPONENTIAL, and RECIPROCAL EXPONENTIAL have originally referred to $a / t_{c}$. In these cases, $t_{c}$ is included in the second fit parameter $k$ since both are constant for a given sample. This is done so that all fit models have the same degree of freedom which increases comparability and also eliminates the need to measure $t_{c}$. The inclusion of $t_{c}$ furthermore would introduce an additional source of error. In contrast, for $\mathrm{GAO}_{1}$ and $\mathrm{GAO}_{2}$, the coating thickness is used as an additional free fit parameter to conform to the requirement of two fit parameters.

Additional fit models described by Menčík et al. [4] and Doerner and Nix [9] were not considered due to their unsuitable curve progression.

These models could not adequately describe the QCSM results of our samples; thus, we propose a new fit model for an improved extrapolation. It is based on the linear additive form Eq. (1) with $E_{I T, 1}=$ $E_{I T, c}$ and henceforth labeled SigmoID.

The Oliver-Pharr method on its own cannot provide the necessary extrapolation for extracting $E_{I T, c}$. For an overall impression, all introduced fit models are fitted to one sample in Fig. 2. It is apparent that the unique curve progression of every model is resulting in a different goodness of fit. 
Table 1 Considered fit models

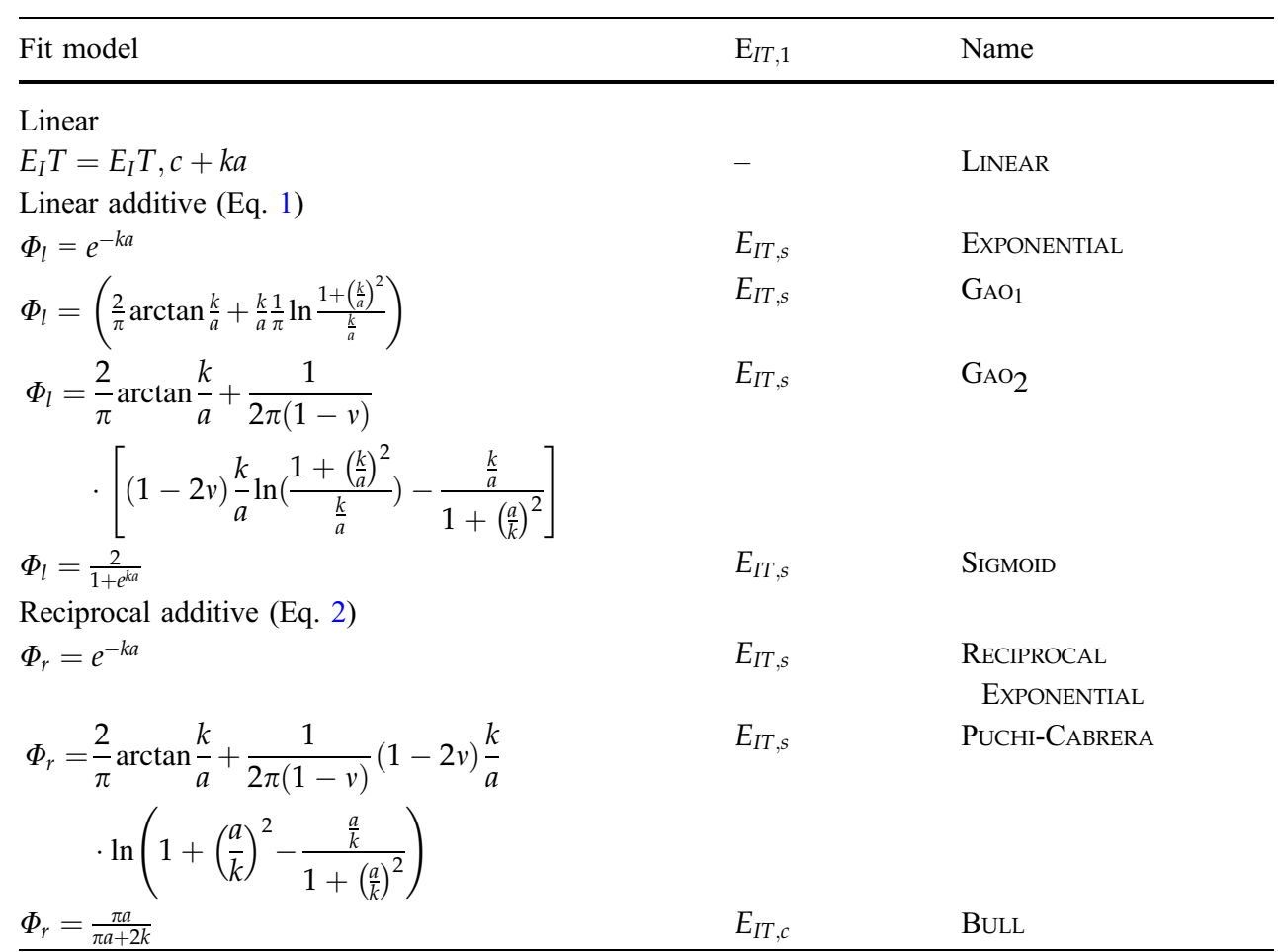

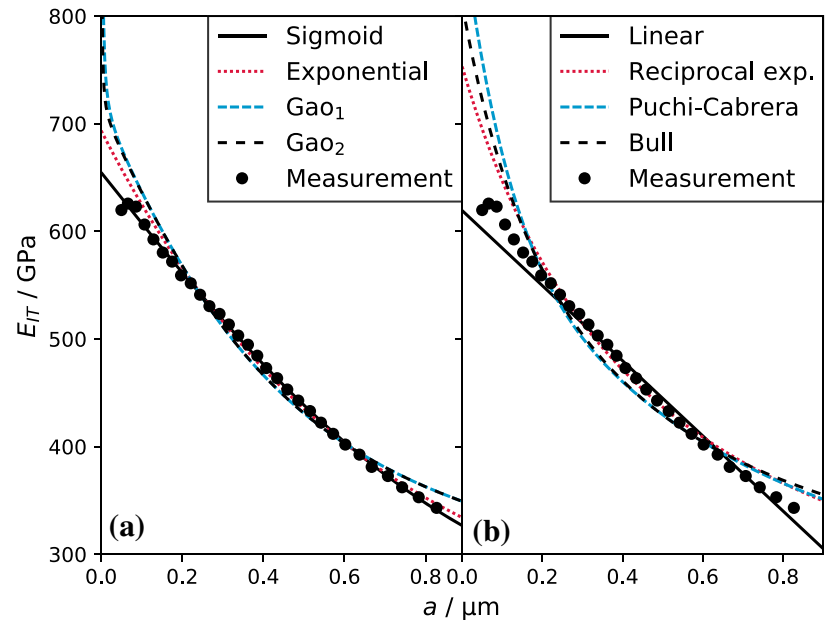

Figure 2 Demonstrative comparison of fit for all introduced fit models on one sample, separate for linear additive (a) and reciprocal additive models and the linear model (b).

\section{Tetrahedral amorphous hydrogen-free} carbon

Hydrogen-free tetrahedral amorphous carbon (ta-C) is a form of amorphous carbon (sometimes called diamond-like carbon (DLC)) with a high degree of $\mathrm{sp}^{3}$-hybridized atoms exceeding $50 \%$. The degree of $\mathrm{sp}^{3}$-hybridized atoms determines properties such as density, $H_{I T}$, and $E_{I T}$ [10], making ta-C the hardest type of amorphous carbon. ta-C coatings can be produced by physical vapor deposition techniques that provide high ionization of the carbon. The coatings investigated in this study were deposited by an industry-scale laser-induced pulsed vacuum arc technique (LaserArc) [11, 12]. This method allows to vary the degree of $\mathrm{sp}^{3}$ hybridization and thus coating properties in a wide range of $20-80 \mathrm{GPa} H_{T T}$. In addition to its outstanding mechanical properties, taC coatings show a beneficial tribological behavior (low friction, low wear) that causes its application as friction-reducing coatings and/or wear protection coating in highly stressed parts, i.e., in automotive applications $[13,14]$. The high $E_{I T}$ of ta-C results in strongly curved $E_{I T}-a$ curves when deposited on conventional steel substrates as shown in Fig. 3.

\section{Materials and methods}

\section{Samples}

All samples were prepared by coating polished disks with ta-C in a Laser-Arc-PVD process. These disks were made of hardened low-alloy chromium steel (100Cr6, EN 1.3505, approximately HRC60). Measurements were conducted on 55 different coating batches. The coatings ranged from $t_{c}=0.7$ to $25 \mu \mathrm{m}$ and 


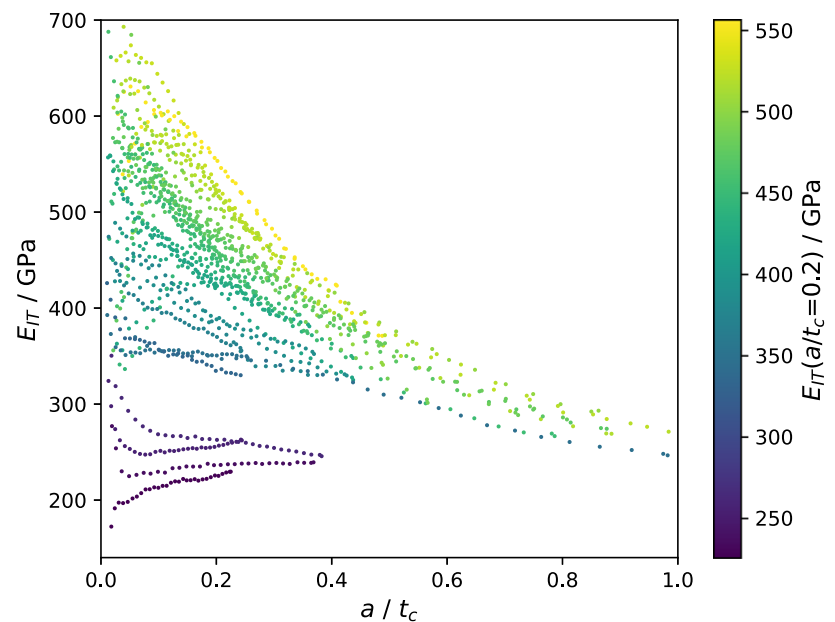

Figure 3 Depth-dependent $E_{I T}$ curves for all considered samples. The curves are colored by their corresponding $E_{I T}$ at a defined $a / t_{c}$; to account for the common measurement errors at lower depths, a comparison value at $a / t_{c}=0.2$ has been chosen.

indentation hardness $H_{I T}=13$ to $74 \mathrm{GPa}$. The roughness of ta-C coatings is influenced by their thickness, so the sample surfaces were polished lightly (typically $\mathrm{Ra}<20 \mathrm{~nm}$ ) for comparable measurements.

\section{Nanoindentation}

All nanoindentation measurements were performed on a nanomechanical testing system Zwick/Roell ZHN using a Berkovich indenter with a nominal tip rounding of less than $200 \mathrm{~nm}$. The device was used in QCSM mode with 30 points of measurement during loading, $40 \mathrm{~Hz}$ frequency, and $100 \mathrm{mN}$ maximum load. The area function was calibrated at least every three samples. The $v$ for ta- $C$ was assumed to be 0.19 [15]. Every sample measurement is comprised of the mean of at least ten individual measurements excluding outliers identified by the device software.

\section{Fit model comparison criteria}

For comparing the fit models, we chose two criteria whose relevance will be outlined as follows:

- Goodness of fit,

- Stability of fit.

\section{Goodness of fit}

Due to the quantity of different fit models, a preselection of the considered fit range could not be made.
Instead, the models were evaluated over several $a / t_{c}$ ranges. The total size of the fit range was fixed at 0.25 . This range was established empirically as a good compromise between as many measurement points as possible from each individual measurement, while including as many different samples as possible, where measurement with large contact radius becomes the limit. Starting at a lower limit of $a / t_{c}=0$ and a corresponding upper limit of $a / t_{c}=0.25$, the fit range was moved incrementally over the whole curve and a new $E_{I T, c}$ calculated for every sample and fit model. Examples are given in Fig. 4. To compare the fit models, for every fit range the mean $\left\langle\chi_{\text {red }}^{2}\right\rangle$ over all samples was calculated with a custom-written software tool for all fit model-fit range combinations.

\section{Stability of fit}

For minimizing the operator influence, the extrapolated $E_{I T, c}$ should be stable for a wide range. Particularly, small changes of the number and position of the measuring points should not cause larger changes in the extrapolated value. Equivalent to the approach for evaluating the goodness of fit, the stability of fit was examined over a variable fit range. For every sample and fit model, the fit range-dependent coefficient of variation $c_{v}\left(E_{I T, \mathcal{c}}\right)$ was calculated. Then, the mean of $c_{v}\left(E_{I T, c}\right)$ over all samples was calculated to produce the mean coefficient of variation $<c_{v}\left(E_{I T, c}\right)>$.

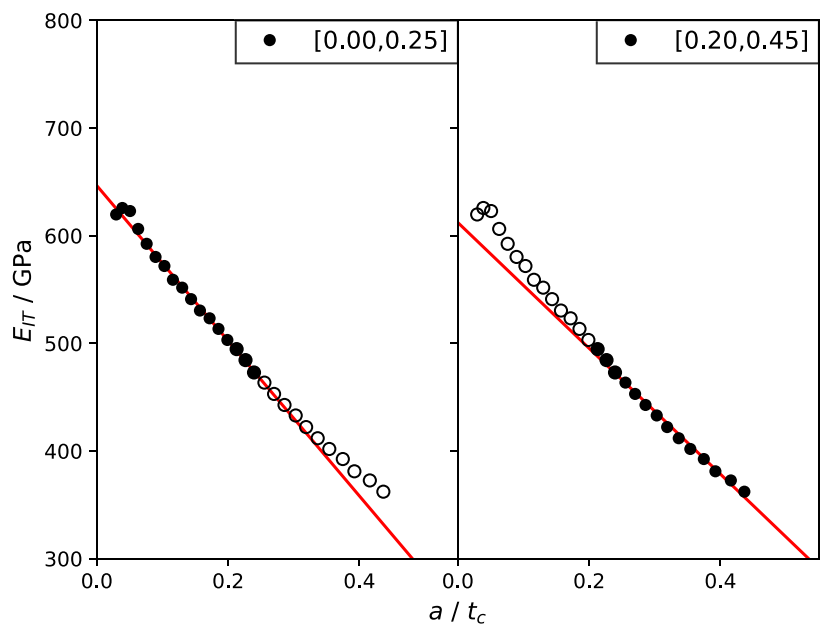

Figure 4 LinEAR fits on a measurement exemplifying the definition of fit ranges with fits from [0, 0.25] and [0.2, 0.45], respectively. 


\section{Results and discussion}

\section{Comparison of fit models}

\section{Goodness of fit}

$<\chi_{\text {red }}^{2}>$ is shown in Fig. 5 for all fit models up to $[0.25,0.5]$. The proposed SIGMOID shows the best fit, with LINEAR showing an equally good fit from $[0,0.25]$ to $[0.1,0.35]$. ExPONENTIAL shows a comparable, albeit slightly worse fit over all fit ranges compared to SIGMOID. Despite its linear character which cannot follow the curved progression, LINEAR performed better than most other models. This is surprising but can be explained by these other models being unsuited for our ta-C samples. Fit ranges with an upper bound higher than 0.5 were not considered due to the unreasonably high load necessary for thick or hard samples to reach this threshold. Out of 55 measurements, only 11 exceeded $a / t_{c}=0.5$.

\section{Stability of fit}

The calculated $\left\langle c_{v}\left(E_{I T, c}\right)\right\rangle$ values for every fit model are listed in Table 2. Due to the fit guidance from $E_{I T, S}$, most models showed a reasonably good stability of fit. Still, there were occasional instabilities for smaller amounts of fitted points which manifested themselves in a considerable higher $E_{I T, c}$ extrapolation compared to the higher fit regions and therefore higher $\left\langle c_{v}\left(E_{I T, c}\right)>\right.$. This is demonstrated in Fig. 6 . Only LiNEAR, SIGMOID, and ExPONENTIAL were able to

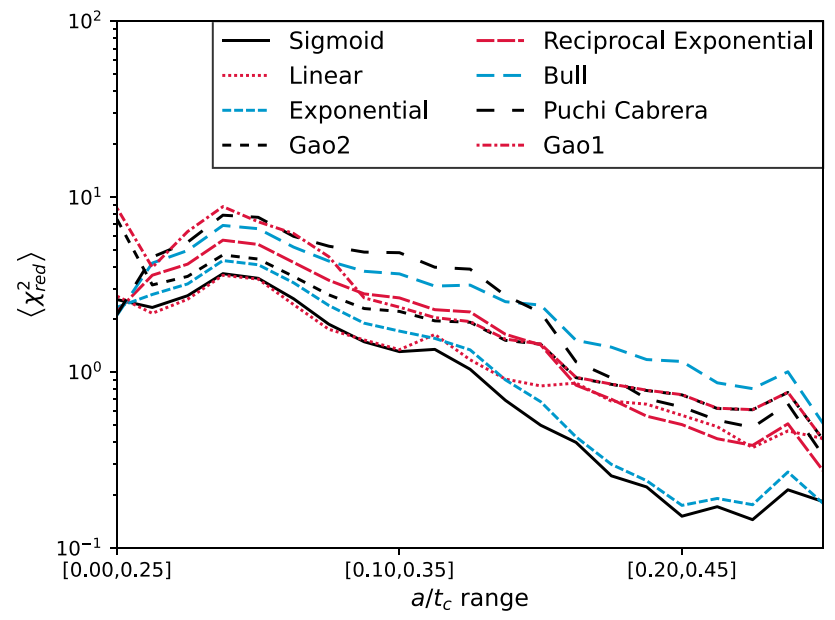

Figure 5 Comparison of goodness of fit for all researched fit models. Lower $\left\langle\chi_{\text {red }}^{2}>\right.$ means better fit. Values for RECIPROCAL EXPONENTIAL are out of bounds.
Table 2 Comparison of stability of fit for the researched fit models. A lower $\left\langle c_{v}\left(E_{I T, c}\right)\right\rangle$ means lower variation of the extrapolated $E_{I T, c}$ when varying the fit boundaries which implies a more stable fit

\begin{tabular}{ll}
\hline Fit model & $<c_{v}\left(E_{I T, c}\right)>$ \\
\hline SIGMOID & 0.08 \\
LINEAR & 0.07 \\
EXPONENTIAL & 0.10 \\
RECIPROCAL EXPONENTIAL & 0.19 \\
$\mathrm{GAO}_{1}$ & 0.72 \\
$\mathrm{GAO}_{2}$ & 0.90 \\
BULL $_{\text {PUCHI-CABRERA }}$ & 3.41 \\
\hline
\end{tabular}

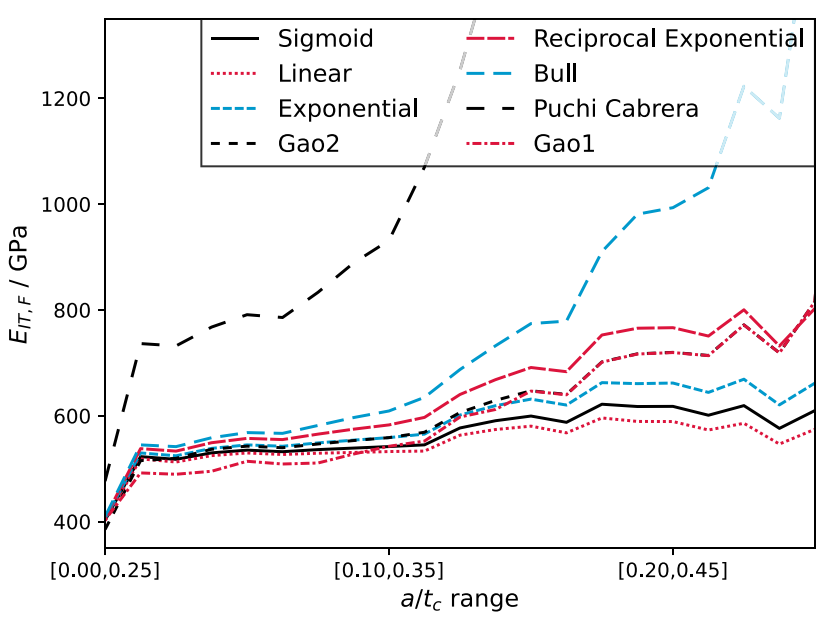

Figure 6 Comparison of extrapolated $E_{I T, c}$ for all considered fit models.

extrapolate a stable $E_{I T, c}$ over all ranges. Accordingly, these had equally the lowest $\left\langle c_{v}\left(E_{I T, c}\right)>\right.$ with RECIPROCAL EXPONENTIAL trailing closely behind. All other fit models' $<c_{v}\left(E_{I T, C}\right)>$ were at least four times higher. RECIPROCAL EXPONENTIAL's instability described by Menčík [4] could not be confirmed.

\section{Correlation of coating thickness}

The SIGMOID fit factor $k$ is connected to $t_{c}$ since it describes the fit curve steepness. The thinner the measured coating, the higher the fitted curve's slope (and thus $k$ ) since it approaches the substrate value more quickly. For thin coatings, it approaches fast, leading to a large $k$. For thick coatings, $E_{I T}$ approaches $E_{I T, S}$ much slower, corresponding to a small $k$. This relationship is particularly strong for ta-C, 
which shows only little disruptive plastic deformation. $k$ as a function of $t_{c}$ is shown in Fig. 7 for the investigated samples. Because of the assumed inverse correlation of fit factor and coating thickness, a fit curve according to Eq. (5) is proposed.

$k=\frac{c}{t_{c}}$

$c$ describes a coating thickness factor specific to the coated system and amounts to $3.139 \pm 0.021$ for the investigated ta-C coatings on steel. Accordingly, with known $k$ and $c$ one can calculate a measured coating's thickness with Eq. (6).

$$
\begin{aligned}
t_{\text {ccalc }}= & \frac{3.139}{k} \Delta t_{\text {ccalc }}=0.021 \frac{1}{k} \\
& +\Delta k \frac{3.139}{k^{2}}
\end{aligned}
$$

This relation enables the determination of coating thickness by evaluating QCSM measurements with the sigmoid fit model. The resulting errors for the whole sample set employing Eq. (6) are shown in Fig. 8. The relative error $\Delta t_{c} c a l c / t_{c}$ calc is consistently smaller than $20 \%$ for $t_{c}$ thinner than $2 \mu \mathrm{m}$. The fitting error can be improved by increasing $h$ with higher $F_{\max }$.

\section{Conclusions}

This work introduces the SIGMOID fit model for the extrapolation of the coating indentation modulus $E_{I T, c}$ and evaluates this model along with several other

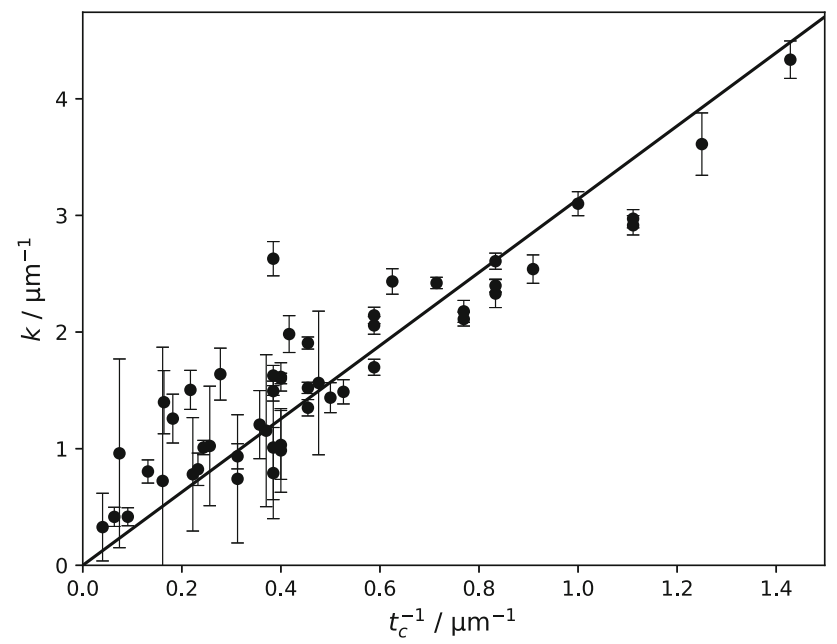

Figure $7 k-t_{c}^{-1}$ plot for ta-C coatings on steel derived from QCSM with fit curve according to Eq. (5).

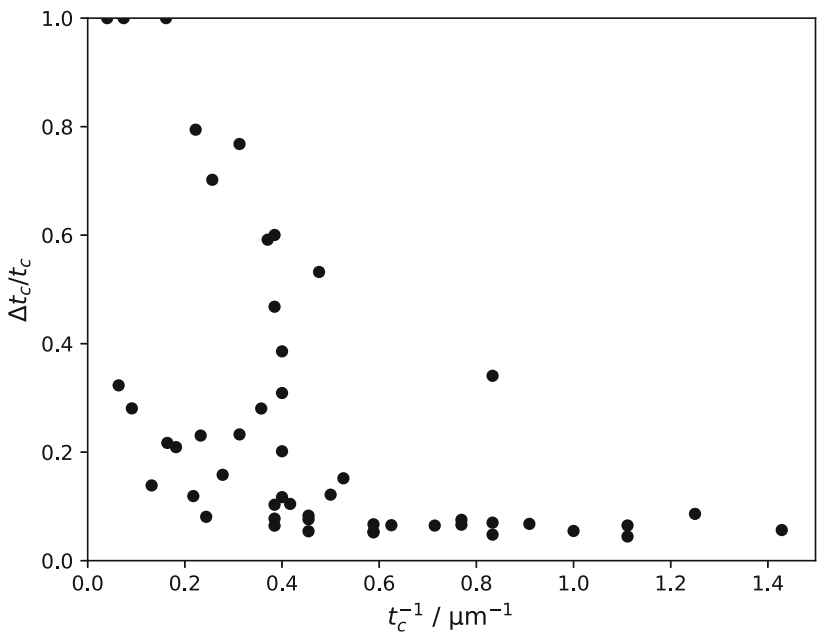

Figure 8 Relative coating thickness error from fitting $\Delta t_{c}$ calc $/ t_{c}$ calc over inverse coating thickness from crater grinding $t_{c}^{-1}$.

models compiled from the literature. For this, a broad data basis was established by measuring 55 steel samples coated with ta-C with $t_{c}=0.7$ to $25 \mu \mathrm{m}$ and $E_{I T, c}=130$ to $740 \mathrm{Gpa}$ with the QCSM method. All methods were evaluated with an in-house software tool regarding goodness of fit and stability of fit. The resulting assessment is given in Table 3 . The newly proposed SIGMOID fit model has the best fit quality with reduced influence of the considered fit range. SIGMOID and LINEAR can be recommended for different use cases, and an assessment is given in Table 4 .

In addition, an empirical approach is demonstrated which allows for a nondestructive coating thickness estimate from depth-resolved $E_{I T}$ curves. This method is applicable even for small samples and complex geometries and is best used for thinner coatings since the error grows with $t_{c}$. While the method is demonstrated for ta-C coatings, other coating systems would require the determination of a

Table 3 Fit model comparison; + positive assessment; negative assessment

\begin{tabular}{lll}
\hline Model & Goodness of fit & Stability of fit \\
\hline SIGMOID & ++ & ++ \\
LINEAR & + & ++ \\
EXPONENTIAL & ++ & ++ \\
RECIPROCAL EXPONENTIAL & - & - \\
$\mathrm{GAO}_{1}$ & -- & -- \\
$\mathrm{GAO}_{2}$ & -- & -- \\
PUCHI-CABRERA & -- & -- \\
BULL & -- & -- \\
\hline
\end{tabular}


Table 4 Fit model recommendation

new correlation factor with the outlined method. Additionally, the correlation can be used to confirm fit correctness of a SIGMOID fit to a ta-C QCSM curve by comparing correlated and expected thickness.

\section{Funding}

Open Access funding enabled and organized by Projekt DEAL.

\section{Declarations}

Conflict of interest The authors declare that they have no conflict of interest.

Open Access This article is licensed under a Creative Commons Attribution 4.0 International License, which permits use, sharing, adaptation, distribution and reproduction in any medium or format, as long as you give appropriate credit to the original author(s) and the source, provide a link to the Creative Commons licence, and indicate if changes were made. The images or other third party material in this article are included in the article's Creative Commons licence, unless indicated otherwise in a credit line to the material. If material is not included in the article's Creative Commons licence and your intended use is not permitted by statutory regulation or exceeds the permitted use, you will need to obtain permission directly from the copyright holder. To view a copy of this licence, visit http://creativecommons.org/licen ses/by/4.0/.

\section{References}

[1] Oliver WC, Pharr GM (2004) Measurement of hardness and elastic modulus by instrumented indentation: advances in understanding and refinements to methodology. J Mater Res 19(01):3-20. https://doi.org/10.1557/jmr.2004.19.1.3
[2] ISO (2016) Metallic materials: instrumented indentation test for hardness and materials parameters-part 4: test method for metallic and non-metallic coatings

[3] Oliver WC, Pethica JB (1989) Method for continuous determination of the elastic stiffness of contact between two bodies

[4] Menčík J, Munz D, Quandt E, Weppelmann ER, Swain MV (1997) Determination of elastic modulus of thin layers using nanoindentation. J Mater Res 12(09):2475-2484. https://doi. org/10.1557/JMR.1997.0327

[5] Huajian G, Cheng-Hsin C, Jin L (1992) Elastic contact versus indentation modeling of multi-layered materials. Int $\mathrm{J}$ Solids Struct 29(20):2471-2492. https://doi.org/10.1016/00 20-7683(92)90004-D

[6] Antunes JM, Fernandes JV, Sakharova NA, Oliveira MC, Menezes LF (2007) On the determination of the young's modulus of thin films using indentation tests. Int J Solids Struct 44(25-26):8313-8334. https://doi.org/10.1016/j.ijsols tr.2007.06.015

[7] Puchi-Cabrera ES, Staia MH, Iost A (2015) A description of the composite elastic modulus of multilayer coated systems. Thin Solid Films 583:177-193. https://doi.org/10.1016/j.tsf. 2015.02.078

[8] Bull SJ (2014) A simple method for the assessment of the contact modulus for coated systems. Phil Mag 95(16-18):1907-1927. https://doi.org/10.1080/14786435.20 14.909612

[9] Doerner MF, Nix WD (1986) A method for interpreting the data from depth-sensing indentation instruments. J Mater Res 1(04):601-609. https://doi.org/10.1557/JMR.1986.0601

[10] Ferrari AC (2004) Diamond-like carbon for magnetic storage disks. Surf Coat Technol 180-181:190-206. https://doi.org/ 10.1016/j.surfcoat.2003.10.146

[11] Scheibe HJ, Schultrich B, Drescher D (1995) Laser-induced vacuum arc (laser arc) and its application for deposition of hard amorphous carbon films. Surf Coat Technol 74-75:813-818. https://doi.org/10.1016/0257-8972(95)0828 $0-8$

[12] Kaulfuss F, Weihnacht V, Zawischa M, Lorenz L, Makowski S, Hofmann F, Leson A (2021) Effect of energy and temperature on tetrahedral amorphous carbon coatings deposited by filtered laser-arc. Materials. https://doi.org/10.3390/ma 14092176 
[13] Kennedy M, Hoppe S, Esser J (2014) Lower friction losses with new piston ring coating. MTZ Worldwide 75(4):24-29. https://doi.org/10.1007/s38313-014-0135-7

[14] Kano M (2015) Overview of DLC-coated engine components. In: Cha SC (ed) Coating technology for vehicle applications. Springer, Cham

[15] Chudoba T (2017) High resolution lateral force-displacement measurements as a tool for the determination of lateral contact stiffness and Poisson's ratio. Surf Coat Technol 330:17-25. https://doi.org/10.1016/j.surfcoat.2017.09.065

Publisher's Note Springer Nature remains neutral with regard to jurisdictional claims in published maps and institutional affiliations. 